\title{
Problematic Trends in Observation Status for Children's Hospitals
}

\author{
David C Synhorst, MD, MPA, Jessica L Bettenhausen, MD*
}

Department of Pediatrics, Children's Mercy Kansas City, University of Missouri-Kansas City School of Medicine, Kansas City, Missouri.

rase

wo children who presented to emergency departments in different cities were diagnosed with diabetes mellitus and ketoacidosis. On presentation, both had significant anion gap metabolic acidosis. Because the patients were deemed unsafe for discharge, the admitting physician placed orders that dictated hospital care, including an order that designated the stay as observation (OBS) or inpatient (IP) status. During their stay, both patients received care including continuous infusion of insulin, intravenous fluids, and frequent lab monitoring. Each child recovered quickly and was discharged in less than 48 hours.

Despite both patients receiving comparable care, recovering well, and being discharged home after a similar length of stay, their encounter designation may be different. Although the patient outcome is of utmost importance, the consequences of labeling an encounter as OBS or IP status are complex and may impact the financial standing of patients, hospitals, and payors. Determining the trajectory of OBS status and its utilization in the pediatric population is vital to understanding the consequences of this designation.

In this issue of the Journal of Hospital Medicine, Tian et $\mathrm{al}^{1}$ describe the increase in OBS status hospitalizations between 2010 and 2019, with OBS stays accounting for approximately one-third of pediatric hospitalizations within children's hospitals in 2019. The increase in OBS status use was described in 19 of 20 of the most common All Patient Refined Diagnosis Related Groups, with the highest growth noted for surgical conditions and diabetes mellitus. ${ }^{1}$ These frequently seen, highstakes conditions, when expertly managed, may result in a safe discharge within 48 hours of admission, but the labor-intensive technical skills to ensure patient safety and high-value care often differ greatly from the idea of simply "observing" a patient.

The scope creep of OBS status in pediatrics is evident. OBS status was initially designed to acknowledge a prolonged outpatient period of monitoring with a goal of determining whether inpatient hospitalization was warranted. However, in most circumstances, the care of children under OBS status differs little from those under IP status; OBS status patients are usually

\footnotetext{
*Corresponding Author: Jessica L Bettenhausen, MD;

Email: jlbettenhausen@cmh.edu; Telephone: 816-802-1493;

Twitter: @jessbetten.
}

Received: July 13, 2021; Revised: July 25, 2021; Accepted: July 26, 2021

(C) 2021 Society of Hospital Medicine DOI 10.12788/jhm.3694 cared for in the same wards and by the same providers as IP status patients. The similarities in care lead to nearly equivalent hospital costs for IP and OBS stays. ${ }^{2}$ Comparable hospital costs would be less concerning if reimbursement were proportional, but OBS status hospitalizations are reimbursed at lower outpatient rates. ${ }^{3}$ The combination of similar costs and lower reimbursement results in a financial liability for children's hospitals.

Tian et al add to the growing body of literature that underscores concerns with OBS stays. ${ }^{1}$ Its increasing use over the past decade represents a troubling continuation of increased OBS status use described by Macy et $\mathrm{al}^{3}$ nearly a decade ago, and the variability with which it is applied suggests that the designation has little connection to the clinical status of patients. Instead, its use is more likely influenced by local payor contracts, individual state laws, and provider culture. For individual institutions, this differential application affects more than just reimbursement. OBS stays are often excluded from nationally representative administrative databases, which makes hospital benchmarking, research on outcomes, and accurate comparison of patient populations impossible. ${ }^{4,5}$

The trends described by Tian et al $^{1}$ raise concerns about the potential impact that OBS stays have on patients and hospital systems across the country. OBS status was created to serve a clinical need, but its inconsistent use places hospitals and the children they treat at risk. This erratic application of OBS status and the serious results of its assignment to pediatric hospitalizations provide evidence that criteria for OBS need to be standardized or otherwise abandoned outright.

Disclosures: The authors reported no conflicts of interest.

Funding: Supported by internal funds of Children's Mercy Kansas City.

\section{References}

1. Tian $Y$, Hall M, Ingram M-CE, Hu A, Raval MV. Trends and variation in the use of observation stays at children's hospitals. J Hosp Med. 2021;16(11):645-651. https://doi.org/10.12788/jhm.3622

2. Fieldston ES, Shah SS, Hall M, et al. Resource utilization for observation-status stays at children's hospitals. Pediatrics. 2013;131(6):1050-1058. https:// doi.org/10.1542/peds.2012-2494

3. Macy ML, Hall M, Shah SS, et al. Pediatric observation status: are we overlooking a growing population in children's hospitals? J Hosp Med. 2012;7(7):530-536. https://doi.org/10.1002/jhm.1923

4. Synhorst DC, Hall M, Harris M, et al. Hospital observation status and readmission rates. Pediatrics. 2020;146(5):e2020003954. https://doi.org/10.1542/ peds.2020-003954

5. Gay JC, Hall M, Morse R, Fieldston ES, Synhorst D, Macy ML. Observation encounters and length of stay benchmarking in children's hospitals. Pediatrics. 2020;146(5):e20200120. https://doi.org/10.1542/peds.2020-0120 